\title{
Conceptual versus procedural approaches to ordering fractions
}

\author{
Lynda R. Wiest ${ }^{1}$ and Frank O. Amankonah ${ }^{2}$ \\ ${ }^{1}$ College of Education, University of Nevada, Reno, USA \\ ${ }^{2}$ Mathematics Department, Great Basin College, Winnemucca, Nevada, USA \\ For correspondence: wiest@unr.edu
}

\begin{abstract}
This paper reports the performance of 30 rising seventh-grade girls on a task in which they were asked to order four fractions from least to greatest. Less than three-fifths attained correct answers. The performance gap was widest between students who attended Title I schools and those who did not, the latter being much more likely to attain correct answers. The achievement gap was less prominent by race/ethnicity, family socioeconomic status, and community type (suburban/urban versus rural). Participants tended to use conceptual and procedural approaches equally, but conceptual approaches were more successful. The most common conceptual strategy was making drawings that illustrated part-whole concepts, and the most common procedural strategy was converting fractions to equivalent fractions. The most problematic fractions to place in order of relative size were the two middle fractions, which were somewhat closer to each other in size than other adjacent pairs and were farthest from the benchmarks of 0 or 1. Based on these and other research findings, we conclude that it would benefit students to possess a greater repertoire of specific strategies, especially conceptual strategies such as use of number lines, benchmarks, and set models, for working with fractions.
\end{abstract}

Keywords: Fractions, Mathematics Performance, Mathematics Strategies, Middle School, Student Differences

\section{Introduction}

Knowledge of fractions is foundational to many areas of mathematics learning, including algebra, proportional reasoning, and probability (Clarke \& Roche, 2009; Fennell, 2007; Siegler \& Pyke, 2013; Strother, Brendefur, Thiede, \& Appleton, 2016). One important skill is an ability to determine the relative size of fractions, which involves comparing the magnitude of two fractions to decide whether they are equivalent or which is greater or less, or ordering three or more fractions from smallest to largest or vice versa.In the United States, comparing fraction size appears in the Common Core State Standards for grades three and four (National Governors Association Center for Best Practices \& Council of Chief State School Officers, 2010), and it continues to be an important mathematics skill thereafter.

In this paper we report the performance of 30 girls who completed the sixth grade and entered the seventh grade about six weeks later. (These girls are hereafter called rising seventh graders.) The girls were asked to order four fractions from least to greatest as part of a larger assessment of various mathematics skills. The girls' overall performance and the approaches they took (conceptual versus procedural) are reported. These data are further analyzed according to four demographic sub groupings (race/ethnicity, family socioeconomic status, school-level socioeconomic status, and community type categorized as urban/suburban versus rural). Finally, participant performance is interpreted in relation to existing literature on fraction knowledge, and additional speculation about the results and suggestions for future research are offered. 


\section{Review of Related Literature}

\section{Student Challenges in Learning Fractions}

Fennell (2007) points out that "fractions are a student's first introduction to abstraction in mathematics" (p. 3). Despite the importance of fraction knowledge, students struggle to learn fraction concepts, including fraction comparison (Clarke \& Roche, 2009; Pantziara \& Philippou, 2012; Siegler \& Pyke, 2013; Sprute \& Temple, 2011; Strother et al., 2016). For example, on the 2007 National Assessment of Educational Progress, only half of U.S. eighth-grade students correctly chose the ordered set 2/7, 1/2, and 5/9as that which appeared from least to greatest from among five multiple choice options of three fractions each(Institute of Education Sciences, 2007).Further, a calculator was available for this item.

Some factors that contribute to students' weak performance with fractions include inappropriate transfer of whole-number ideas to fractions (e.g., larger numbers mean greater magnitude) and a focus on individual fraction components (numerator, denominator) rather than a fraction as a single entity (Clarke \& Roche, 2009; Lamon, 2012; Siegler \& Pyke, 2013). Further, fraction ideas become more complex when comparing the magnitude of fractions that appear in different representations. Pantziara and Philippou (2012)state, "The process of abstraction starts when students realize that different sharing situations can end up in equivalent fractions" (p. 66).They note that of five fraction sub constructs, students perform most poorly on that of "measure," in which students identify a fraction as a point on a number line.The ability to locate a fraction on a number line is important to understanding the relative size of fractions, which in turn helps students view a fraction as a single number; this suggests a need for greater use of the number line for fraction investigations (Fennell, 2007; Pantziara \& Philippou, 2012; Sprute \& Temple, 2011). Nevertheless, students tend to have difficulty with fraction placement on a number line, and the measure sub construct appears in mathematics textbooks and instruction less frequently than the dominant part-whole sub construct (Pantziara \& Philippou, 2012).

One key source of struggle with fraction magnitude is weak knowledge of the role of the numerator and denominator. Students might gauge fraction magnitude by the size of either the numerator or the denominator rather than integrating the two meaningfully (Meert, Grégoire, \& Noël, 2010; Siegler \& Pyke, 2013; Sprute \& Temple, 2011), and whole-number interference can lead students to think larger denominators mean larger fractions (Pantziara \& Philippou, 2012). Fractions with common numerators cause more problems than those with common denominators (Meert et al., 2010), and fractions with different numerators and denominators can be especially challenging (Lamon, 2012).Further, students might employ a "gap-thinking" strategy in which they compare the absolute difference between a numerator and denominator of one fraction to that of another to determine relative size (Clarke \& Roche, 2009). Clearly, inappropriate whole-number generalizations undergird some of these issues. Finally, among other potential problems that arise in working with fractions is a distance effect whereby it is more difficult to distinguish fractions (as well as whole numbers) that are closer together than those that are farther apart (Sprute \& Temple, 2011). The degree to which these difficulties are conceptual (based on the learner's ability to understand the concepts) versus instructional is uncertain (Sprute \& Temple, 2011).

\section{Role of Conceptual and Procedural Knowledge}

Although conceptual knowledge, or meaning- and relationship-oriented knowledge, is often emphasized as being particularly important in mathematics in comparison with procedural knowledge, or operational (e.g., algorithmic) knowledge of mathematics, many educators and researchers note that both are important to successful mathematics learning (Gabriel et al., 2012; Hallett, Nunes, \& Bryant, 2010; Pantziara \& Philippou, 2012). Pantziara and Philippou (2012) state:

Learners relying on conceptual knowledge develop sophisticated mathematical thinking, 
while those who rely on procedural knowledge face difficulties in handling complicated conceptual structures.... Procedural learning, although indispensable in mathematics... may lead to insufficient understanding and mere completion of routine mathematical tasks. (p. 62) Not only are both conceptual and procedural knowledge necessary for mathematics competence, so too is the relationship between these two types of knowledge (Bergsten, Engelbrecht, \& Kagesten (2017). Gabriel et al. (2012) describe conceptual and procedural knowledge specifically in relation to fractions:

In the domain of fractions, conceptual knowledge refers to a combination of the general properties of rational numbers (such as the principle of equivalent fractions), the understanding of the roles of the numerator and denominator, and the understanding of global fraction magnitudes.... Knowing how to calculate the lowest common denominator to add or subtract fractions with different denominators is a prime example of procedural knowledge. (p. 138)

The distinction between using conceptual and procedural approaches seems to be particularly apparent in relation to fractions (Hallett et al., 2010). Based on research conducted with sixth graders, Siegler and Pyke (2013) suggest that understanding magnitudes, and related tasks such as ordering several fractions according to size, "appears to be a particularly important aspect of conceptual understanding of fractions" (p. 1995) and correlates with mathematics achievement in general. They say this conceptual knowledge is important for developing associated procedural knowledge.

Clark and Roche (2009) conducted individual interviews with more than 11,000 students ages 4-8 in Australia to investigate mental strategies used to identify the larger of two fractions. The two most successful strategies students used were classified as conceptual approaches. A residual thinking strategy involved determining the amount needed to build up to a whole, as in recognizing that one more eighth is needed to get from seven-eighths to one whole. The other strategy involved use of benchmarks in which the two fractions were compared to a third "anchor" fraction such as one-half or sometimes the whole number one to help determine fraction magnitude in order to aid comparison.

\section{Suggested Instructional Approaches for Improving Fraction Understanding}

Conceptual understanding of fractions, including knowledge of the relative size of fractions, requires development of strong fraction sense, or a good "feel" for fractions that helps students "make appropriate connections, determine size, order, and equivalence, and judge whether answers are or are not reasonable" (Lamon, 2012, p. 136), often acquired through a substantial amount of appropriate informal experiences. This includes using key fraction benchmarks, such as zero, onehalf, and one, to solve or aid in solving various types of fraction problems. Based on their work with third-grade students, Bray and Abreu-Sanchez (2010) conclude, "This strategy of comparing to onehalf not only is more efficient than finding common denominators or using cross multiplication for the problem posed, but also requires conceptual and relational ways of thinking about fractions" (p. 91). Meert et al.'s (2010) findings from research with fifth and seventh graders indicate that assessing fraction magnitude involves attention to the fraction as a whole, as well as its components. Accordingly, it is imperative that students learn the meaning of the numerator and denominator and their relationship to each other in a holistic or unified manner (Clarke \& Roche, 2009; Siegler \& Pyke, 2013). Some instructional strategies recommended for improving students' understanding of fractions are those that allow students to: use and make visual representations of fractions; partition objects and drawings into equal-sized pieces; visualize fraction concepts (e.g., physical and pictorial representations); see fractions as a single entity and locate them on a number line that includes whole numbers; develop more than one strategy for solving fraction tasks, including number-sense approaches such as use of benchmarks; and use these approaches in active, engaging, collaborative 
endeavors (Bray \& Abreu-Sanchez, 2010; Gabriel et al., 2012; Lamon, 2012; Pantziara \& Philippou, 2012; Sprute \& Temple, 2011).

\section{Summary and Rationale for This Paper}

Both teachers and students struggle in the teaching and learning of fractions (e.g., Clarke \& Roche, 2009). As noted, fraction magnitude and relative size are among the specific areas of difficulty. These important areas are given less instructional time than part-whole concepts. Further, Meert et al. (2010) note, "The representation of fraction magnitude has been tested by only a few studies, all of them testing adults" (p. 245). Therefore, in this paper we examine student performance on a single fraction problem from various angles in order to contribute to the body of knowledge on students' ability to order fractions by relative size.

\section{Research Purpose and Questions}

The purpose of this investigation was to examine rising seventh-grade girls' performance on ordering fractions. Specifically, we sought to assess girls' performance in terms of accuracy in ordering four fractions from least to greatest and the broad methods (conceptual or procedural) used to do so. For exploratory purposes, we further examined the girls' performance by the demographic variables of race/ethnicity, community type (urban/suburban versus rural), and socioeconomic status (SES) measured at both the family and school levels.

The research questions for this study were:

1. What degree of achievement do rising seventh-grade girls demonstrate in ordering four fractions?

2. Do rising seventh-grade girls tend to use conceptual or procedural methods to order fractions, and which is more successful?

3. Do rising seventh-grade girls' achievement and solution strategies in ordering fractions tend to differ by race/ethnicity, community type, or family or school socioeconomic status?

\section{Method}

This investigation involved examination of a single problem included within a brief assessment of rising seventh-grade girls' performance on several mathematics problems involving different mathematics topics. The girls completed the assessment at the beginning of a residential math-andtechnology summer camp.

\section{Participants}

Girls who solved the fraction problem examined in this paper were a diverse group who attended a six-day residential mathematics-and-technology camp during the summer midway between the sixth and seventh grades (rising seventh graders).The 30 participants were from across Northern Nevada. Of these, $22(73.3 \%)$ were from urban/suburban communities and $8(26.7 \%)$ were from rural communities. In terms of race/ethnicity, $21(70.0 \%)$ were White, $3(10.0 \%)$ were Hispanic/Latina, 2 (6.7\%) were Black/African American, 2 (6.7\%) were American Indian/Alaska Native, and 2 (6.7\%) were of mixed race/ethnicity. Free and reduced-price lunch status served as a proxy for family SES, and school type classified as Title I or non-Title I represented school SES. In terms of SES, 17 (56.7\%) girls did not indicate free/reduced-price lunch status, whereas $13(43.3 \%)$ did provide such evidence, respectively here considered medium/high SES and low SES, and 19 (63.3\%) girls had not attended a Title I school the previous year, whereas $11(36.7 \%)$ had attended a Title I school. Family SES differed from school SES for 18 (60\%) of the girls. In other words, only 12 participants (40\%) either had lowSES indicators for both family and school (free/reduced-price lunch status and Title I school) or for neither of these. Because the girls came from a wide variety of geographic locations and family 
circumstances, the seasoned teachers who served as camp instructors reported that the girls' ability levels and background experiences were much broader than that of a "typical" class for their grade level.

\section{Assessment Item}

The assessment item participants solved was: Order the fractions from least to greatest: 1/1, 1/3, 5/8, 1/12. Participants solved the problem individually on paper and did not have access to a calculator while solving the problem. As noted earlier, comparing fractions is listed as a standard in the Common Core State Standards (CCSS) for grades three and four mathematics and thus should have been addressed and built on for several years by the time these girls solved the indicated problem. The state in which participants reside (Nevada) adopted the CCSS in 2010.

\section{Data Analysis}

In addition to scoring the problem as correct or incorrect, the general approach used to answer the problem was classified as conceptual, procedural, or indeterminate. A conceptual approach involves presenting information in a meaningful, relational way, such as demonstrating why or how something works or making sense of it in a real-world context, whereas a procedural approach involves executing standard steps to solve a task, as in implementing an algorithm (e.g., Hallett et al., 2010; Tularam \& Hulsman, 2013). Problem solutions classified as conceptual in this study included those that involved making part-whole drawings, writing a meaning-oriented explanation (e.g., smaller denominators indicate larger parts), or determining the proximity of fractions to benchmarks(e.g., one-half and one).Approaches considered procedural included problems solved by converting fractions to decimals through division or to equivalent fractions with a common denominator. Finally, problem solutions that could not be classified because students simply wrote an answer without showing work were labeled "indeterminate" due to these mental approaches providing no "window" into the strategy used. (Examples of student work using conceptual and procedural approaches may be found in Figures 1 and 2 of the Results section.)

To classify each student's solution into one of the three approaches, the two authors met and jointly developed the meaning of the three solution-strategy options noted above. They then co-scored and agreed on the first five students' problem solutions before coding the final 25 students' work independently. Of the 25 problems classified independently, the two raters agreed on $24(96 \%)$. A third mathematics educator then coded the problem that involved disagreement, after which the two authors discussed that input and agreed on the final classification to be used for that problem. The first author independently categorized the specific approaches participants used (e.g., use of a drawing or converting fractions to equivalent fractions).

Due to the low number of participants in this study, no statistical analyses were conducted. For example, the number of items in each cell of a two-by-two contingency table comparing correct and incorrect performance according to solution strategy chosen (conceptual, procedural) ranged from 3 to 9. Although a one-sample chi-square test would generally be an appropriate method to statistically analyze this type of result, the results are typically only considered reasonably accurate if the expected frequencies are five or greater (Green \& Salkind, 2014; Lowry, 2013), which was not the case here. Therefore, we report the results as numbers and percents to be examined for exploratory purposes only.

\section{Results}

Students' solutions are first categorized into conceptual versus procedural approaches, with some being unclassifiable ("indeterminate") due to use of mental approaches. Participant success in solving the problems is reported for the sample as a whole and for selected demographic groups. Specific 
strategies students used within the broader categories of conceptual and procedural approaches are also discussed.

\section{Conceptual Versus Procedural Approaches}

Of the 30 participants who completed the problem, $13(43.3 \%)$ used procedural approaches, 12 $(40.0 \%)$ used conceptual approaches, and $5(16.7 \%)$ used indeterminate mental methods. More than half of all participants attained a correct answer for the problem, but the proportion was highest for those who used conceptual methods. (See Table 1.)

Table 1. Problem accuracy by major solution strategy

\begin{tabular}{lcc}
\hline Solution Strategy & \multicolumn{2}{c}{ Accuracy } \\
\cline { 2 - 3 } & Correct & Incorrect \\
\hline Conceptual & $8(66.7 \%)$ & $4(33.3 \%)$ \\
Procedural & $7(54.0 \%)$ & $6(46.0 \%)$ \\
Indeterminate (Mental) & $2(40.0 \%)$ & $3(60.0 \%)$ \\
Totals & $17(56.7 \%)$ & $13(43.3 \%)$ \\
\hline
\end{tabular}

The success rate for solving the problem ranged among demographic groups from $73.7 \%$ for students who did not attend Title I schools to $27.3 \%$ for students who attended Title I schools. The difference between these two school SES categories was the widest among demographic groups assessed, with groups formed by race/ethnicity, family SES, and community type being separated by narrower margins, as shown in Table 2. Among the three demographic groups with smaller performance gaps, racial minorities collectively outperformed White students, and students from low family SES backgrounds were somewhat more successful than students of middle/high family SES, as were urban/suburban students compared with rural students. Students more likely to use conceptual solution strategies were White (compared with collective racial minorities), middle/high (versus low) family SES, from a non-Title I (versus Title I) school, and urban/suburban (compared with rural). (See Table 2.)

Table 2. Problem accuracy and major solution strategy by demographic group

\begin{tabular}{cllllllll}
\hline \multicolumn{6}{c}{ Race/Ethnicity } & \multicolumn{2}{c}{ Family SES } & \multicolumn{2}{c}{ School SES } & \multicolumn{2}{c}{ Community Type } \\
\hline & White & $\begin{array}{l}\text { Racial } \\
\text { Minority }\end{array}$ & $\begin{array}{l}\text { Middle/ } \\
\text { High }\end{array}$ & Low & $\begin{array}{l}\text { Non- } \\
\text { Title I }\end{array}$ & Title I & $\begin{array}{l}\text { Urban/ } \\
\text { Suburban }\end{array}$ & Rural \\
\hline Number/Percent Correct & & & & & & & \\
& $11 / 21$ & $6 / 9$ & $9 / 17$ & $8 / 13$ & $14 / 19$ & $3 / 11$ & $13 / 22$ & $4 / 8$ \\
& $(52.4 \%)$ & $(67.7 \%)$ & $(52.9 \%)$ & $(61.5 \%)$ & $(73.7 \%)$ & $(27.3 \%)$ & $(59.1 \%)$ & $(50.0 \%)$ \\
Solution Strategy & & & & & & & & \\
Conceptual & $9 / 21$ & $3 / 9$ & $8 / 17$ & $4 / 13$ & $8 / 19$ & $4 / 11$ & $10 / 22$ & $2 / 8$ \\
& $(42.9 \%)$ & $(33.3 \%)$ & $(47.1 \%)$ & $(30.8 \%)$ & $(42.1 \%)$ & $(36.4 \%)$ & $(45.5 \%)$ & $(25.0 \%)$ \\
Procedural & $8 / 21$ & $5 / 9$ & $5 / 17$ & $8 / 13$ & $8 / 19$ & $5 / 11$ & $9 / 22$ & $4 / 8$ \\
Indeterminate & $(38.1 \%)$ & $(55.6 \%)$ & $(29.4 \%)$ & $(61.5 \%)$ & $(42.1 \%)$ & $(45.5 \%)$ & $(40.9 \%)$ & $(50.0 \%)$ \\
(Mental) & $4 / 21$ & $1 / 9$ & $4 / 17$ & $1 / 13$ & $3 / 19$ & $2 / 11$ & $3 / 22$ & $2 / 8$ \\
& $(19.0 \%)$ & $(11.1 \%)$ & $(23.5 \%)$ & $(7.7 \%)$ & $(15.8 \%)$ & $(18.2 \%)$ & $(13.6 \%)$ & $(25.0 \%)$ \\
\hline
\end{tabular}

*Because percents were rounded to the nearest tenth, rounding error may occur that causes the three percents for a demographic group to not equal $100 \%$ exactly. 


\section{Specific Solution Strategies}

Of the 15 correct answers that showed a specific solution strategy, the two most common approaches were converting the fractions to equivalent fractions (a procedural approach) and making a drawing (a conceptual approach). Specifically, 6 involved converting the fractions to equivalent fractions with a common denominator ( 3 of these did so only for two of the four fractions, $1 / 3$ and 5/8), and 5 involved making a drawing of rectangles and/or circles partitioned into equal parts with some shaded. In one case, a student drew both four circles and four rectangles. In another drawing the four rectangles drawn were different sizes, with larger rectangles being used for fractions with larger denominators, yet the student appeared to use size of the shaded portion relative to the whole to compare fractions and arrive at an answer. One other correct method involved the use of benchmarks whereby the student designated $1 / 1$ as being equal to one and then mentally determined whether each of the other three fractions was less than or more than one-half. Another correct method involved converting the fractions to decimals. The remaining two accurate methods were only partially classifiable and tended to use a mix of methods across the fractions in the problem. Figure 1 shows sample student work for correctly applied conceptual and procedural approaches.

Figure 1. Successful participant approaches to ordering four fractions from least to greatest
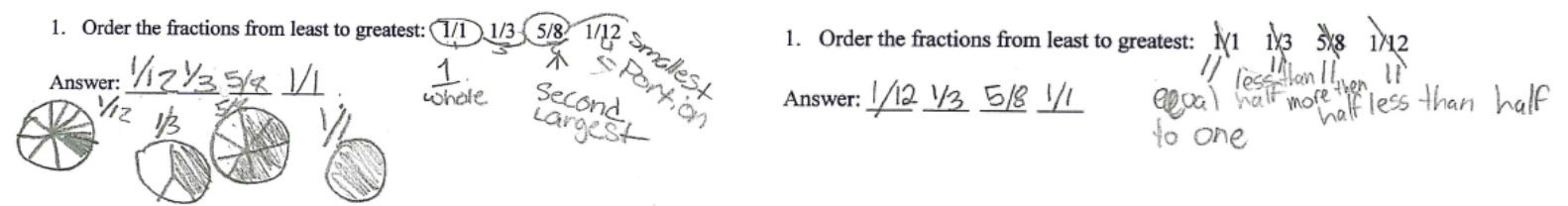

Conceptual approaches yielding correct answers (partitioned, shaded region models and benchmarks, respectively).
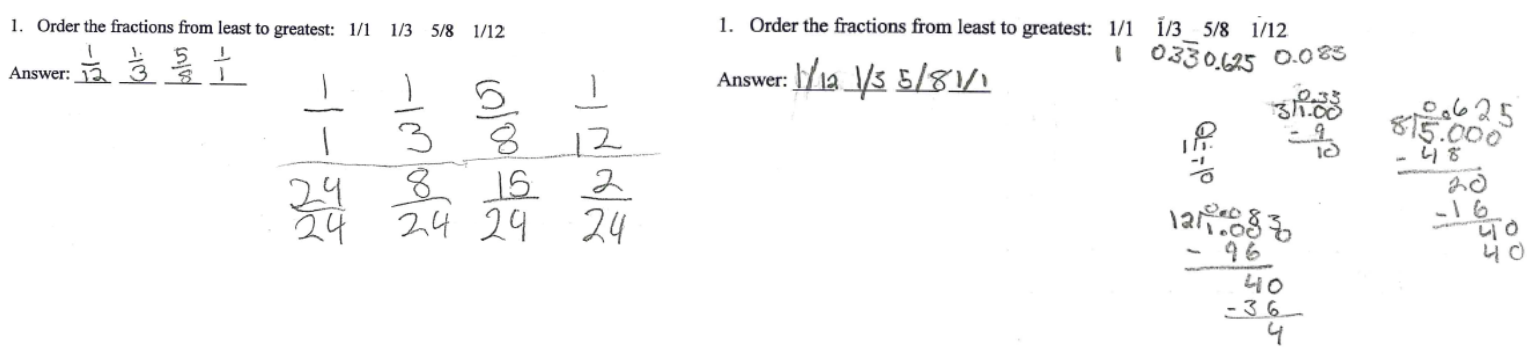

Procedural approaches yielding correct answers (conversion to equivalent fractions and to decimals, respectively).

Of the 13 problems with incorrect answers, the most common error, occurring 9 times (69\%), was to reverse the order of the middle two fractions $(1 / 3,5 / 8)$. The next most common error for incorrect answers, which appeared 3 times (23\% of answers), was to reverse the order of all four fractions and present the answer from greatest to least. No pattern appeared in the ten incorrect problems that showed specific strategies, with one or two students each converting the fractions to decimals, using gap thinking, inaccurately conceptualizing fraction magnitude, cross multiplying, using a drawing, and converting only one of the four fractions to an equivalent fraction that was insufficient to attain a correct answer. The student who made an error in using drawings used circles for two fractions and rectangles for the other two. She reversed the order of the middle two fractions in the answer, one of which was drawn as a circle and the other as a rectangle. One student who inaccurately conceptualized the problem wrote, "The smaller the piece the lesser chance it has of being the greatest," indicating a probable focus on denominators only rather than numerator and denominator as an integrated whole. The student who got the problem wrong after converting the four fractions to decimals made a computation error that resulted in converting $1 / 12$ to 0.83 instead of 0.083 . Figure 2 shows student work for incorrect or incorrectly applied conceptual and procedural strategies. 
1/12 "has the smallest pieces" might have determined the fraction's overall magnitude mentally. Another who seemed to acknowledge that piece size mattered did not integrate its meaning into the fraction's magnitude as a whole and thus did not solve the problem correctly: "The smaller the piece the lesser chance it has of being the greatest." Similarly, the other student who produced an incorrect answer wrote, "The smaller the fraction [likely meaning the denominator] the less peices [sic]." None of these students gave direct evidence of blending the numerator and denominator into a meaningful single numeric value.

\section{Discussion}

Overall, student performance on this fraction task tends to mirror student performance in other research studies (e.g., Institute of Education Sciences, 2007)in that students do not show strong performance comparing and ordering fractions. Students used conceptual and procedural approaches equally, but those using conceptual approaches were somewhat more successful in attaining a correct answer. This aligns with the perspectives of some authors and researchers who consider conceptual approaches particularly important, while acknowledging the key role of procedural skills (e.g., Hallett et al., 2010; Siegler \& Pyke, 2013). One concern with procedural approaches is whether students try to make conceptual sense of their work. For example, one participant rightly converted all four fractions in this task to decimals. However, she made an error on one, attaining 0.83 instead of 0.083 for $1 / 12$. The fact that she did not notice this major mistake is a lingering potential issue with procedural strategies.

Similar to previous research, students tended to use a limited number of success-oriented approaches, in particular, use of equivalent fractions and drawings. In 6 of the 25 cases where strategies were identifiable, participants used efficient methods by using drawings or conversions to decimals or equivalent fractions only for those fractions that seemed to challenge them the most (here, typically $1 / 3$ and 5/8). Drawings used as aids to solve the problem exclusively employed part-whole models, which have been found to dominate mathematics textbooks (Pantziara \& Philippou, 2012). Partitioning visual (e.g., area) models is considered an important aid to comparing fractions, although student difficulties with doing so have been noted (Lamon, 2012). In order to determine relative size of the four fractions in the problem posed in this investigation, no student attempted to locate the fractions on a number line or to use a set model, and only one student used benchmarks by comparing the fractions to one-half or one.

Three students appeared to know that denominator size relates to fraction magnitude, but they tended not to integrate that information with numerator size to form a single value. This problematic focus on individual fraction components has been noted elsewhere (e.g., Lamon, 2012; Siegler \& Pyke, 2013). Two students used the inaccurate and unsuccessful approach termed "gap thinking" that has been found in other studies (Clarke \& Roche, 2009). This approach involves comparing the difference between the numerator and denominator of each fraction. Focusing on fraction parts separately and the gap between them can indicate inappropriate reference to whole numbers rather than understanding the meaning of a fraction as a single entity.

The most common error in answers as presented, regardless of specific strategy used, was to reverse the order of the middle two fractions but correctly place the first and last. This fits Sprute and Temple's (2011) finding of the distance effect in which problem solvers tend to be able to order fractions that are farther apart in magnitude more successfully than those that are closer to each other. However, the middle two fractions were not greatly different in size compared with other adjacent pairs, so participants might have found it easier to place the most extreme fractions, the two closest to 0 and 1 , than the two in the middle. Another consideration is that the first and last fractions in the problem statement appear in reverse order in the correct answer. One or more students who 
noticed that might have thought the answer was thus simply the reverse order of the original problem. Of course, two fractions in which both the numerators and denominators differ and are not multiples of each other are among pairs that are more difficult to compare in size (Clarke \& Roche, 2009). Similarly, no students used the effective strategy known as residual thinking, whereby students determine the amount needed to build up to a whole (cf. Clarke \& Roche, 2009). Perhaps this is because this strategy has not been fostered in classroom work with fractions or because no fractions in this task evoked this method, as in two fractions that are each one part away from a whole (e.g., 3/4 and 4/5). Thus, the specific fractions used in a task can influence student performance. The less frequent error in which three students presented the answer in reverse order could be due to students misreading or not attending to the problem directions, which required the answer to appear in leastto-greatest order.

Taken together, overall performance on this task seems to indicate that students are more likely to achieve correct results when using conceptual approaches or when applying procedural methods that they understand or are well rehearsed. It also appears that students could benefit by developing a greater range of strategies for comparing the relative size of fractions. In particular, use of number lines, benchmarks, and set models appear to be used infrequently in textbooks and classroom instruction but could benefit students by expanding their repertoires for comparing fractions (Bray \& Abreu-Sanchez, 2010; Clarke \& Roche, 2009; Lamon, 2012; Pantziara \& Philippou, 2012; Sprute \& Temple, 2011). All of these methods can promote conceptual thinking, including development of fraction sense, which is highly important to student success in working with fractions (Lamon, 2012).We thus contend that while student difficulties with fractions might at times be conceptual (internal to individual students), instructional approaches used for developing fraction ideas carry especially heavy weight in terms of student understanding and performance.

Student performance on this fraction problem might lend support to the idea that school SES is more influential in a student's education than family SES, which runs counter to findings by Chiu (2010). (Both were influential in Chiu, but family SES more so. Also see Brown-Jeffy, 2009, regarding schoollevel effects on student performance.)Comparisons of performance by race/ethnicity, family SES, school SES, and community type were most discrepant between participants who did and did not attend a Title I school. If this result holds true for studies with larger samples, it would generate both concern about the educational differences between these two types of schools but also hope that if we can identify and improve the critical aspects of Title I schools that might influence student learning, we can develop better educational experiences in those institutions that might supersede student demographic factors that influence academic performance. We find it surprising that 18 of the 30 participants in this investigation had low family SES or attended a Title I school, but not both. (Note that this number could differ slightly if some families were eligible but chose not to apply for free/reduced-price lunch.) Although it is less surprising that urban/suburban students were somewhat more successful than rural students, it is atypical that racial minorities outperformed White students and students from low family SES backgrounds were somewhat more successful than students with higher SES backgrounds. It is uncertain whether this is because the students who attend the summer camp already have some degree of interest and/or success in mathematics, making them less reflective of the population at large, or whether the individuals from groups who tend to exhibit lower performance in mathematics did not presume successful performance and thus took the task more seriously and exerted more effort and mindfulness. Finally, for all four demographic-group pairs examined, the collective group that typically shows greater mathematics achievement (White, middle/high family SES, non-Title I school, urban/suburban community residence; see, for example, Brown-Jeffy, 2009 and Chiu, 2010) was more likely to use conceptual solution strategies. This would be worth pursuing further to determine whether this finding holds across larger research samples. If so, it would highlight the importance of expecting all students, including underperformers, to develop and use conceptual approaches to mathematics. Note that 
suburban students typically outperform both urban and rural students (e.g., Graham \& Provost, 2012), but suburban was combined with urban here because most urban students in this sample reside in a medium-sized city well served by university programs and other services that are much less available to rural students in the state. Interestingly, however, although suburban students tend to show greater achievement than urban or rural students overall, Graham and Provost (2012) did not find such differences in the West, a finding worth pursuing more fully to determine why this might be so.

\section{Limitations and Suggestions for Future Research}

One limitation of this investigation is that it was based on a single problem and thus presents minimal data and an inability to explore the results of comparing and ordering different fractions. Further, the fact that participant work was analyzed post hoc with few students writing their thought processes and students not explaining their strategies orally as they solved the problem limited insight into student thinking about the investigated problem, in particular, for those who used mental methods and only wrote an answer to the problem. Finally, the participants were girls who chose to attend a girls' mathematics and technology camp. Although the group was fairly diverse with a broad set of personal and educational experiences, as well as varied ability levels, this group cannot be considered representative of girls this age in general. Participants in this study might possess stronger mathematics skills or more confidence, effortful behavior, persistence, and/or out-of-school support than other girls their age.

Due to the importance of fraction knowledge in mathematics education, continued research should be conducted on student understanding of fractions to create an increasingly nuanced picture. Many variables are worth investigating in addition to student accuracy in solving problems. General approaches used (conceptual, procedural, or a combination of the two), as well as specific strategies, should be identified and compared to accuracy in solving fraction problems. Mental approaches might also be investigated, with students explaining orally or in writing how they arrive at answers. Instructional context (teaching methods and materials) should be examined to determine how it relates to students' solution methods and degree of accuracy in solving fraction problems. Selected other variables worth considering in terms of relationship to student performance are the number of fractions ordered (e.g., three versus four), the specific types of fractions used (same denominator or numerator, denominators that are multiples of each other, fractions that are varying distances from each other and from benchmark fractions/numbers, fractions that are less than and greater than one, etc.), and whether or not calculator use is permitted. Finally, the performance of various demographic groups formed by gender, race/ethnicity, family and school SES, and so forth should be examined in relation to student performance in determining the relative size of fractions.

\section{References}

Bergsten, C., Engelbrecht, J., \& Kagesten, O. (2017). Conceptual and procedural approaches to mathematics in the engineering curriculum-Comparing views of junior and senior engineering students in two countries. EURASIA Journal of Mathematics Science and Technology Education, 13(3), 533-553.

Bray, W. S., \& Abreu-Sanchez, L. (2010). Using number sense to compare fractions. Teaching Children Mathematics, 17(2), 90-97.

Brown-Jeffy, S. (2009). School effects: Examining the race gap in mathematics achievement. Journal of African American Studies, 13(4), 388-405.

Chiu, M. M. (2010). Effects of inequality, family and school on mathematics achievement: Country and student differences. Social Forces, 88(4), 1645-1676.

Clarke, D. M., \& Roche, A. (2009). Students' fraction comparison strategies as a window into robust understanding and possible pointers for instruction. Educational Studies in Mathematics, 72(1), 127-138.

Fennell, F. (2007, December). Fractions are foundational. NCTM News Bulletin, p. 3. Retrieved from: http://www.nctm.org/about/content.aspx?id=13316

Gabriel, F., Coché, F., Szucs, D., Carette, V., Rey, B., \& Content, A. (2012). Developing children's understanding of fractions: An intervention study. Mind, Brain, and Education, 6(3), 137-146. 
Graham, S. E., \& Provost, L. E. (2012). Mathematics achievement gaps between suburban students and their rural and urban peers increase over time. Durham, NH: University of New Hampshire, Carsey Institute. Retrieved from http://www.carseyinstitute.unh.edu/publications/IB-Graham-Math-Achievement-K-8.pdf

Green, S. B., \& Salkind, N. J. (2014). Using SPSS for Windows and Macintosh: Analyzing and understanding data (7th ed.). Boston: Pearson.

Hallett, D., Nunes, T., \& Bryant, T. (2010). Individual differences in conceptual and procedural knowledge when learning fractions. Journal of Educational Psychology, 102(2), 395-406.

Institute of Education Sciences. (2007). National Assessment of Educational Progress (NAEP): 2007 Mathematics Assessment. Washington, DC: U.S. Department of Education, National Center for Education Statistics.

Lamon, S. J. (2012). Teaching fractions and ratios for understanding: Essential content knowledge and instructional strategies for teachers (3rd ed.). New York: Routledge.

Lowry, R. (2013). Concepts and applications of inferential statistics. Retrieved from http://vassarstats.net/textbook/index.html

Meert, G., Grégoire, J., \& Noël, M.-P. (2010). Comparing the magnitude of two fractions with common components: Which representations are used by 10- and 12-year-olds? Journal of Experimental Child Psychology, 107(3), 244-259.

National Governors Association Center for Best Practices \& Council of Chief State School Officers. (2010). Common Core State Standards for mathematics. Washington, DC: Authors.

Pantziara, M..\&Philippou, G. (2012). Levels of students' “conception” of fractions. Educational Studies in Mathematics, 79(1), 6183.

Siegler, R. S., \& Pyke, A. A. (2013). Developmental and individual differences in understanding of fractions. Developmental Psychology, 49(10), 1994-2004.

Sprute, L., \& Temple, E. (2011). Representations of fractions: Evidence for accessing the whole magnitude in adults. Mind, Brain, and Education, 5(1), 42-47.

Strother, S., Brendefur, J. L., Thiede, K. \& Appleton, S. (2016). Five key ideas to teach fractions and decimals with understanding. Advances in Social Sciences Research Journal, 3(2), 132-137.

Tularam, G. A., \& Hulsman, K. (2013). A study of first year tertiary students' mathematical knowledge-Conceptual and procedural knowledge, logical thinking and creativity. Journal of Mathematics and Statistics, 9(3), 219-237.

Van de Walle, J. A., Karp, K. S., \& Bay-Williams, J. M. (2013). Elementary and middle school mathematics: Teaching developmentally (8th ed.). Boston: Pearson. 